\title{
A transcription factor to call their own
}

Not to be outdone by the everexpanding list of $\mathrm{T}$ cell subsets and their defining transcription factors, macrophages are let in on the act by new research showing that interferon regulatory factor 5 (IRF5) is a subsetdefining factor for M1 macrophages with both activating and repressive transcriptional functions.

Monocyte-derived macrophages that are differentiated with granulocyte-macrophage colony stimulating factor (GM-CSF) adopt an M1 (classical) phenotype, which is associated with the production of pro-inflammatory cytokines such as interleukin-12 (IL-12). Conversely, differentiation with M-CSF leads to the polarization of M2 (alternative) macrophages that produce antiinflammatory cytokines such as IL-10. This study showed that treatment of human monocytes with GM-CSF but not M-CSF resulted in increased expression of IRF5.
In line with a role for IRF5 in macrophage plasticity, as well as in initial polarization, the conversion of M2 macrophages to M1 macrophages by culture with GM-CSF also resulted in increased IRF5 expression.

Human M2 macrophages that were forced to express IRF5 had increased expression of IL-12p70 and IL-23 and decreased expression of IL-10, whereas inhibition of IRF5 expression in M1 macrophages resulted in the converse levels of cytokine production. These data show that IRF5 promotes the IL-12 ${ }^{\text {hi }}$ IL-23 $3^{\text {hi }}$ IL-10 ${ }^{\text {low }}$ cytokine profile that is associated with M1 macrophages, and further studies showed that this is the result of direct effects on transcriptional activity. The mRNA levels for these cytokines were modulated by IRF5 in a manner consistent with the protein data, and genome-wide expression analysis showed that IRF5 induces

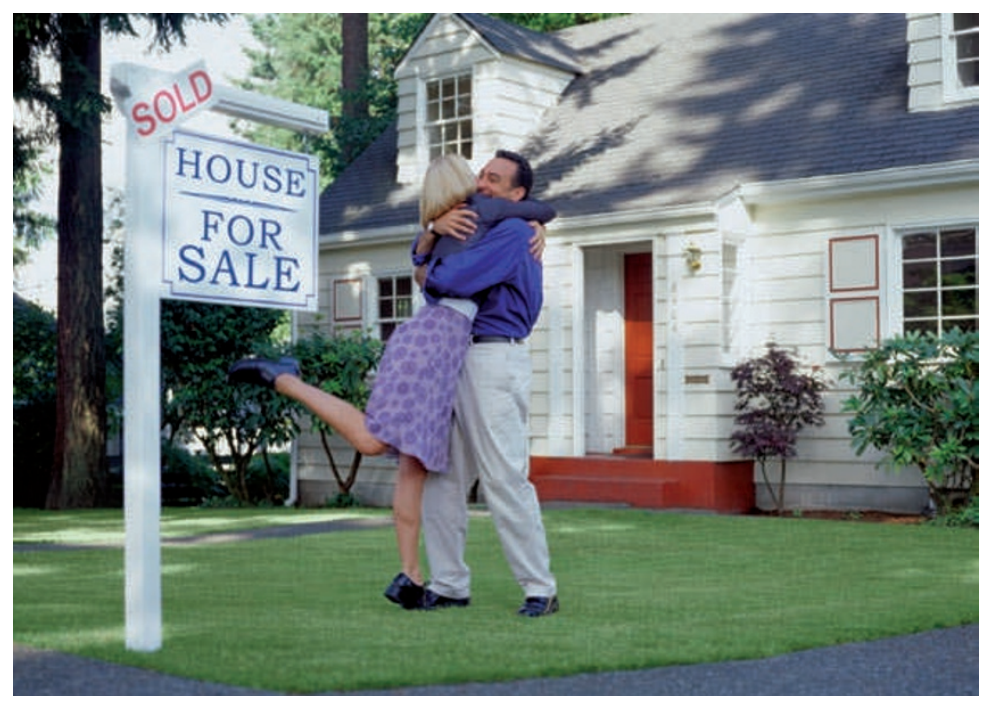

the expression of M1-specific genes and represses the expression of M2-specific genes. Furthermore, IRF5 was shown to bind to the promoter regions of the genes encoding IL-12p40, IL-12p35, IL-23p19 and IL-10.

Recruitment of RNA polymerase II to the IL10 promoter only occurred after the dissociation of IRF5 from this region. This is in keeping with a role for IRF5 in transcriptional inhibition of the IL10 gene, and is supported by the demonstration that IRF5 inhibits the expression of a reporter construct containing the IL10 promoter sequence. Mutations in IRF5 or the IL10 promoter region that disrupted IRF5-DNA binding prevented this inhibitory effect on reporter expression.

These data indicate that the expression of GM-CSF at sites of inflammation can drive M1 macrophage polarization through increased IRF5 expression, which has both positive and negative effects on the transcription of macrophage subset-specific genes. In turn, M1 macrophages are known to drive pro-inflammatory Thelper $1\left(\mathrm{~T}_{\mathrm{H}} 1\right)$ cell responses. Indeed, the forced expression of IRF5 by human M2 macrophages resulted in increased proliferation of MHC-mismatched T cells and the differentiation of both $\mathrm{T}_{\mathrm{H}} 1$ and $\mathrm{T}_{\mathrm{H}} 17$ cell populations.

Kirsty Minton

ORIGINAL RESEARCH PAPER Krausgruber, T. et al. IRF5 promotes inflammatory macrophage polarization and $\mathrm{T}_{\mathrm{H}} 1-\mathrm{T}_{\mathrm{H}} 17$ responses. Nature Immunol. 16 Jan 2011 (doi:10.1038/ni.1990) 